(C) 2020, The Authors. Published by FASS Inc. and Elsevier Inc. on behalf of the American Dairy Science Association ${ }^{\circledR}$. This is an open access article under the CC BY-NC-ND license (http://creativecommons.org/licenses/by-nc-nd/4.0/).

\title{
Genetic correlations between body weight, daily weight gain, and semen characteristic traits in young Norwegian Red bulls
}

\author{
H. B. Olsen, ${ }^{*}$ (D) B. Heringstad, (D) and G. Klemetsdal (1) \\ Department of Animal and Aquacultural Sciences, Faculty of Biosciences, Norwegian University of Life Sciences, PO Box 5003 , 1433 Aas, \\ Norway
}

\section{ABSTRACT}

The aim of this study was to estimate genetic parameters for body weight (BW) at $150 \mathrm{~d}$ (Bw_150d), and $330 \mathrm{~d}$ (Bw_330d) of age and average daily weight gain (Dwg), and to estimate genetic correlations between these traits and semen characteristic traits: volume; concentration (Conc); motility in fresh, 24-h, and 48-h samples (Mot0h, Mot24h, Mot48h); and sperm defects. Data were collected at the performance test station of young Norwegian Red bulls from 2002 to 2012, before selection of bulls for artificial insemination. The weight and growth data consisted of observations for 3,209 bulls, and andrology information was available for up to 2,034 of these bulls. Genetic parameters were estimated using linear animal models. Models for BW and growth traits included the group and year the bull left the station and the pen they occupied during weighing (group-year-pen) and parity of their dam as fixed effects. Models for andrology traits had group-year, age in months (11 to 15$)$, and the interaction between ejaculate number and days since previous collection included as fixed effects. Estimated heritability was 0.14 for Bw_150d, 0.26 for Bw_330d, and 0.34 for Dwg; the estimated genetic correlations among these traits were all favorable. Both BW traits correlated favorably with all the semen characteristic traits (0.20 to 0.76), whereas Dwg was favorably correlated with volume, Mot24h, Mot48h, and sperm defects, and unfavorably correlated with Conc $(-0.25)$ and Mot0h $(-0.53)$. Our results indicate that the genetic correlations between weight and growth traits and semen characteristics depend on the age of the bulls. Although most genetic correlations were favorable, selection for higher daily weight gain between 150 and $330 \mathrm{~d}$ might explain the slight negative genetic trends observed for semen characteristics in young Norwegian Red bulls.

Received December 23, 2019.

Accepted February 27, 2020.

*Corresponding author: henriette.berg.olsen@nmbu.no
Key words: heritability, genetic parameter, andrology

\section{INTRODUCTION}

Olsen et al. (2020) found that semen characteristic traits of young Norwegian Red bulls showed a slightly unfavorable genetic trend between 1994 and 2016 . Thus, phenotypic selection on semen characteristics practiced at the performance test station has not been sufficient to genetically improve semen volume or quality over time. We hypothesized that the unfavorable genetic trends were caused by selection for other traits that have unfavorable genetic correlations with andrology traits. In contrast to the many studies estimating genetic parameters for cow fertility based on very large data sets (e.g., Andersen-Ranberg et al., 2005; Tiezzi et al., 2012; Carthy et al., 2015), genetic studies on bull fertility are few and based on a relatively small number of animals. Further, genetic correlations between sperm quality and performance traits are largely lacking for both dairy and beef cattle (Berry et al., 2014; Thundathil et al., 2016). Regarding BW, growth traits, and semen characteristics, only 2 studies have been published, both of which used data from beef cattle. In these studies, the genetic correlations between BW (weaning weight and yearling weight) traits and semen characteristics (concentration, motility, and percent of normal sperm cells) ranged from -0.36 to 0.75 (Knights et al., 1984; Smith et al., 1989), and genetic correlations between average daily weight gain and motility and percent of normal sperm were -0.36 and 0.34 , respectively (Smith et al., 1989).

Norwegian Red is a dual-purpose breed in which growth is an important trait. Slaughter weight and carcass classification are included in the total merit index (Geno, 2018). Average daily weight gain measured at the performance test station was, until 2013, used as one of the criteria for selection of bulls for AI. Data from the performance test station can therefore be used to meet the objective of this paper; namely, to estimate genetic correlations among BW traits, daily 
weight gain, and semen characteristic traits for young Norwegian Red bulls.

\section{MATERIALS AND METHODS}

\section{Data}

Data from the performance test station for young Norwegian Red bulls from 2002 to 2012 were made available by Geno (Hamar, Norway), the breeding organization for Norwegian Red cattle. Each year, around 250 of the most promising Norwegian Red bull calves were performance tested. Bulls arrived at the station at 4 to 5 mo of age and were placed in pens of 12 to 18 animals by age. Concentrates were given according to age, and grass silage was available ad libitum. Conformation and temperament were assessed during the stay. Further, bulls were weighed, and BW at $150( \pm 5) \mathrm{d}$ and $330( \pm 5)$ d were used to compute average daily weight gain. At the end of the stay, when the bulls were around 12 mo old, several andrology traits were measured and used to ensure that only bulls with acceptable semen quality were selected and sent to the AI center.

Routine registrations of bulls' BW (kg) at age 150 $\mathrm{d}\left(\mathbf{B w} \_\mathbf{1 5 0 d}\right)$ and at $330 \mathrm{~d}\left(\mathbf{B w} \_330 \mathrm{~d}\right)$ and average daily weight gain (Dwg; g/d) from 150 to 330 d from yr 2002 to 2012 were provided by Geno. Records of Bw_330d $<299$ or $>500 \mathrm{~kg}$ were considered erroneous and removed. The same was done for observations of Dwg $<500$ or above $>2,000 \mathrm{~g} / \mathrm{d}$. Bulls were transferred from the station in groups (either for slaughter or to the AI center), and all bulls were assigned with a group number and a group year, as well as the pen number they occupied during weighing. Finally, the data contained information on whether the bull was the dam's first calf.

Andrology data were available for 2,034 of the 3,209 bulls with weight and growth information, and included the following traits:

(1) Volume (mL).

(2) Concentration recorded by photometer (Conc). The photometer could not register measurements $<390 \times 10^{6}$; therefore, concentration was set to $390 \times 10^{6}$ if the photometer read zero but sperm cells were found during microscopic evaluation. Because of this, we categorized Conc into 10 classes: $0,1-390$, thereafter in intervals of 200 , and finally $>1,790 \times 10^{6}$ spermatozoa $/ \mathrm{mL}$.

(3) Motility measured in fresh samples by subjective inspection under a phase contrast microscope (given as percentage of moving sperm cells, with 10-percentage-unit increments starting at
0; Mot0h). After first inspection, samples were stored in a refrigerator until they were reactivated (heated to $38^{\circ} \mathrm{C}$ in $5 \mathrm{~min}$ ) and evaluated for motility again after $24 \mathrm{~h}$ (Mot24h) and $48 \mathrm{~h}$ (Mot48h), measured in the same way as Mot0h.

(4) Sperm defects - a binary trait scored as 1 if more than $10 \%$ of a particular spermatozoa (spz) defect was present in the sample, or $>20 \%$ defects in total, and 0 otherwise. Defects were loose heads, abnormalities in the tail or the intermediate part, distal droplets, and proximal droplets.

Only semen collections with volume $>0 \mathrm{~mL}$ were kept for analyses. Samples with volume $>12 \mathrm{~mL}$ or Conc $>3,000 \times 10^{6} \mathrm{spz} / \mathrm{mL}$ were considered erroneous and removed. Bulls had to be between 320 and $472 \mathrm{~d}$ (10.5$15.5 \mathrm{mo}$ ) old at the test-day, and only bulls that had been assigned group number and group year were kept. See Olsen et al. (2020) for further details on semen collection and editing of data.

Descriptive statistics for the andrology, BW, and growth traits are summarized in Table 1. Figure 1 shows Dwg plotted against Bw_150d and Bw_330d. The pedigree of the bulls was traced back as far as possible and included 41,356 animals in the additive genetic relationship matrix.

\section{Models}

Initially, univariate, linear animal models were run in DMU using the average information (AI)REML procedure (Madsen and Jensen, 2013) to estimate variance components for the body weight traits and Dwg. The following model was fitted:

$$
\mathrm{Y}_{i j k}=\operatorname{gyp}_{i}+\operatorname{pod}_{j}+\mathrm{a}_{k}+\mathrm{e}_{i j k},
$$

where $\mathrm{Y}_{i j k}$ is an observation of Bw_150d, Bw_330d, or Dwg on the $k$ th bull; gyp $_{i}$ is the fixed effect of the $i$ th group-year-pen $(i=1, \ldots, 261)$; $\operatorname{pod}_{j}$ is the fixed effect of the parity of the dam in 2 classes $(j=1$ : dam's first calf, or 2: second or later calf); $a_{k}$ is the random additive genetic effect of the $k$ th bull $N \sim\left(0, \mathbf{A} \sigma_{a}^{2}\right)$, with $\mathbf{A}$ being the additive genetic relationship matrix and $\sigma_{a}^{2}$ the additive genetic variance; and $e_{i j k}$ is the random residual $N \sim\left(0, \mathbf{I} \sigma_{e}^{2}\right)$, where $\mathbf{I}$ is an identity matrix and $\sigma_{e}^{2}$ is the residual variance.

For andrology traits, the following linear animal repeatability model was fitted:

$$
\begin{gathered}
\mathrm{Y}_{i j k l m o}=\operatorname{age}_{i}+\text { group-year }_{j}+\text { collection_n-interval } \\
\\
+t d_{1}+a_{m}+p e_{m}+e_{i j k l m o},
\end{gathered}
$$


Table 1. Descriptive statistics of semen characteristics and BW and growth traits measured on Norwegian Red bulls at the performance test station

\begin{tabular}{|c|c|c|c|c|c|c|}
\hline Trait & $\mathrm{n}$ & Samples (n) & Mean & $\mathrm{SD}$ & Minimum & Maximum \\
\hline Volume $(\mathrm{mL})$ & 7,634 & 2,034 & 2.4 & 1.4 & 0.5 & 12 \\
\hline Conc $^{1}$ & 7,635 & 2,034 & 2.5 & 1.6 & 0 & 9 \\
\hline $\operatorname{Mot}^{2}(\%)$ & 7,364 & 2,014 & 63.8 & 21.9 & 0 & 80 \\
\hline $\operatorname{Mot} 24 \mathrm{~h}^{2}(\%)$ & 4,934 & 1,591 & 60.4 & 20.9 & 0 & 80 \\
\hline $\operatorname{Mot} 4 \mathrm{~h}^{2}(\%)$ & 1,165 & 899 & 52.3 & 25.5 & 0 & 80 \\
\hline Sperm defects ${ }^{3}$ & 7,640 & 2,034 & 0.05 & 0.21 & 0 & 1 \\
\hline Bw_150d ${ }^{4}(\mathrm{~kg})$ & 3,209 & 3,209 & 163.6 & 21.5 & 92.9 & 250 \\
\hline Bw_330d ${ }^{4}(\mathrm{~kg})$ & 3,209 & 3,209 & 411.6 & 29.5 & 299 & 500 \\
\hline $\operatorname{Dwg}^{4}(\mathrm{~g} / \mathrm{d})$ & 3,209 & 3,209 & $1,377.7$ & 118.7 & 850 & 1,811 \\
\hline
\end{tabular}

${ }^{1}$ Concentration categorized into 10 classes: $0,1-390$, thereafter increments of 200 , and finally $>1,790 \times 10^{6}$ spermatozoa $/ \mathrm{mL}$.

${ }^{2}$ Motility in fresh samples (Mot0h) and after storing for $24 \mathrm{~h}$ (Mot24h) and $48 \mathrm{~h}$ (Mot48h).

${ }^{3}$ Binary trait; scored as 1 if $>10 \%$ of the sperm in the sample had a particular defect or $>20 \%$ defects in total, 0 otherwise. Defects were loose heads, abnormalities in the tail or the intermediate part, distal droplets, and proximal droplets.

${ }^{4} \mathrm{BW}$ of bulls when $150 \mathrm{~d}$ (Bw_150d) and $330 \mathrm{~d}$ (Bw_330d) old, and average daily weight gain (Dwg) in between.

where $\mathrm{Y}_{i j k l m o}$ is the oth observation on 1 of 6 andrology traits; $\operatorname{age}_{i}$ is the fixed effect of the $i$ th age class in months $(i=11, \ldots, 15)$; group-year ${ }_{j}$ is the fixed effect of the $j$ th group and year the bull left the test station $(j$ $=1, \ldots, 74)$; collection $\_$n-interval $l_{k}$ is the fixed effect of $k$ th class of ejaculate number (from $1=$ first semen collection to $6=$ the sixth or more collection) and interval in days since previous collection $(1=1$ to $4 \mathrm{~d}, 2=5$ to $10 \mathrm{~d}$, and $3=>10 \mathrm{~d}$ ), $k=1, \ldots, 16$ (not used in the model for Mot48h because the variable was not significant at $P<0.05$ in a proc GLM in SAS). Test-day $(t d)$, additive genetic $(a)$, permanent environmental $(p e)$, and residual ( $e$ ) effects were assumed random; see below for their distributions.

Bivariate linear animal models were used to estimate genetic correlations among the weight and growth traits and between weight and growth traits and semen characteristic traits. The following assumptions were made for the distribution of the random test-day $(t d)$, additive genetic $(a)$, permanent environmental (pe), and residual $(e)$ effects in the bivariate models:

$$
\begin{gathered}
{\left[t d_{\text {andro }}\right] \sim\left(0, \mathbf{I} \times \sigma_{t d_{2}}^{2}\right),} \\
{\left[\begin{array}{c}
a_{\text {weight or growth }} \\
a_{\text {weight, growth, or andro }}
\end{array}\right] \sim N\left(\left(\begin{array}{l}
0 \\
0
\end{array}\right), \mathbf{A} \otimes\left(\begin{array}{cc}
\sigma_{a_{1}}^{2} & \sigma_{a_{1} a_{2}} \\
\sigma_{a_{1} a_{2}} & \sigma_{a_{2}}^{2}
\end{array}\right)\right),} \\
{\left[p e_{\text {andro }}\right] \sim\left(0, \mathbf{I} \times \sigma_{p e_{2}}^{2}\right),} \\
{\left[\begin{array}{c}
e_{\text {weight or growth }} \\
e_{\text {weight, growth, or andro }}
\end{array}\right] \sim N\left(\left(\begin{array}{l}
0 \\
0
\end{array}\right), \mathbf{I} \otimes\left(\begin{array}{cc}
\sigma_{e_{1}}^{2} & \sigma_{e_{1} e_{2}} \\
\sigma_{e_{1} e_{2}} & \sigma_{e_{2}}^{2}
\end{array}\right)\right),}
\end{gathered}
$$

where $\mathbf{A}$ is the additive genetic relationship matrix. The test-day and permanent environmental effects were only included in the model for the andrology traits (andro), where $\sigma_{t d_{2}}^{2}$ is the test-day variance and $\sigma_{p e_{2}}^{2}$ is the permanent environmental variance, and $\mathbf{I}$ are identity matrices. The (co)variance matrices for additive genetic and residual effects contained variances on the diagonal and covariances on the off-diagonal. In the bivariate analysis of weight or growth and andrology traits, the residual covariance was restricted to zero because the measurements differed in both time and space.

Results from the univariate analyses were used to estimate the heritability $\left(h^{2}\right)$ of weight and growth traits, and results from bivariate models between Dwg and semen characteristic traits were used for the andrology traits. The formulas were

$$
\begin{gathered}
h_{\text {weight }}^{2}=\frac{\sigma_{a}^{2}}{\sigma_{a}^{2}+\sigma_{e}^{2}}, \\
h_{\text {andrology }}^{2}=\frac{\sigma_{a}^{2}}{\sigma_{a}^{2}+\sigma_{p e}^{2}+\sigma_{t d}^{2}+\sigma_{e}^{2}} .
\end{gathered}
$$

Standard errors were computed using Taylor series approximation.

\section{RESULTS AND DISCUSSION}

\section{BW and Growth Traits}

Figure 1a shows that there was little or no phenotypic association between Bw_150d and Dwg, probably 
a)

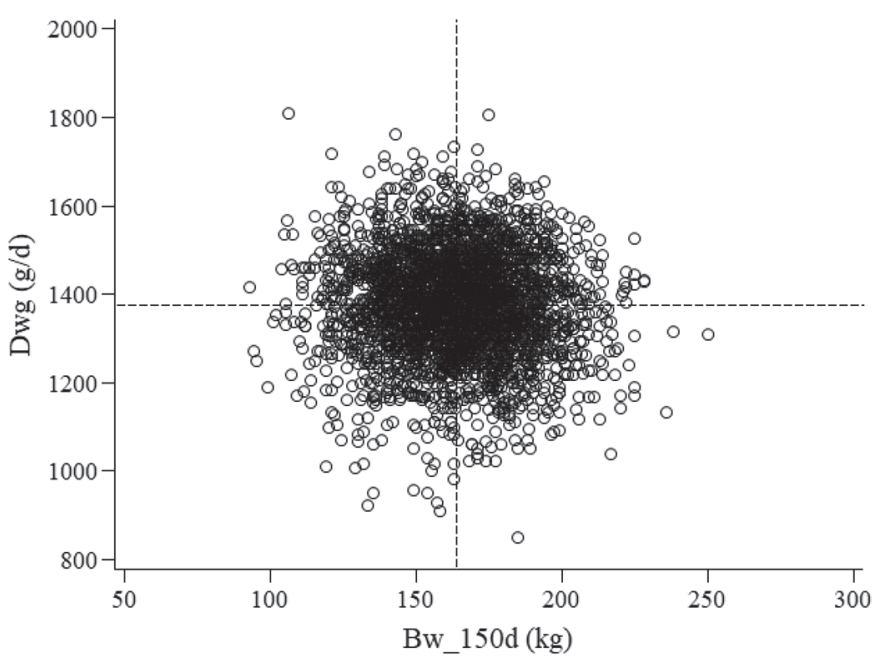

b)

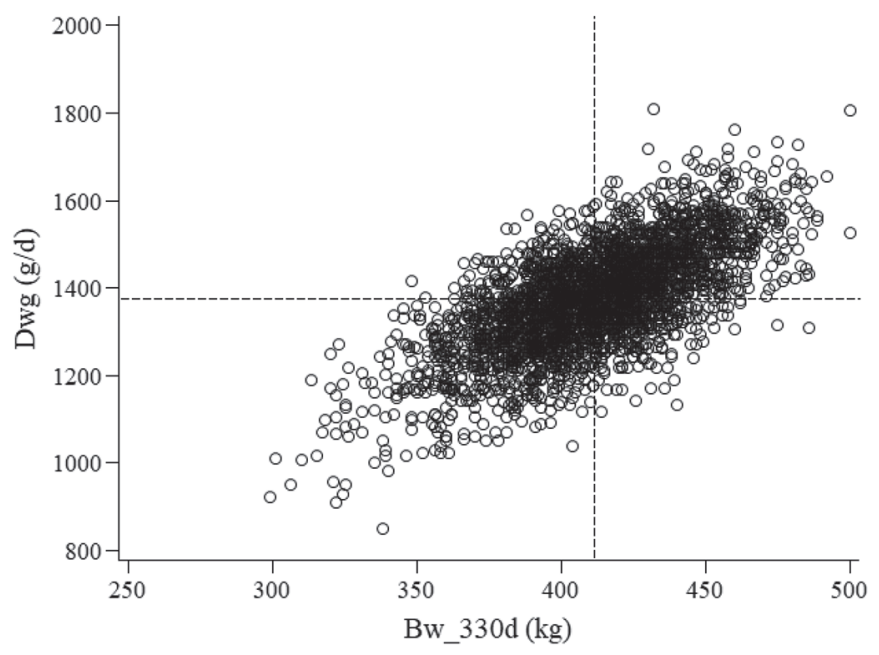

Figure 1. Average daily weight gain (Dwg; g/d) plotted against (a) BW at age $150 \mathrm{~d}$ (Bw_150d) and (b) BW at age $330 \mathrm{~d}$ (Bw_330d) for Norwegian Red bulls $(\mathrm{n}=3,209)$ from 2002 to 2012. Dashed lines show the mean for each trait.

because Bw_150d was mainly affected by the environment before arriving at the test station. However, Bw_330d was phenotypically correlated strongly with Dwg (Figure 1b). Despite the clear positive phenotypic association, the figure also shows that some bulls with below-average weight could grow well above average and vice versa.

Estimated heritability was 0.14 for Bw_150d, 0.26 for Bw_330d, and 0.34 for Dwg (Table 2). The lower heritability estimate for Bw_150d was likely caused by noise from the prestation environment compared with BW measured at the later stage and daily weight gain recorded during the performance test. In a previous study, also using data from the performance test station for Norwegian Red (Aass, 1996), the heritabilities (SE) of Bw_330d and Dwg (from 90 to $330 \mathrm{~d}$ ) were estimated to be $0.33(0.15)$ and $0.30(0.14)$, respectively, which corresponds with our results. Přibyl et al. (2008) estimated genetic parameters for live BW in dual-purpose Czech Fleckvieh bulls at various ages (60 to $400 \mathrm{~d}$ ) and found that heritability decreased slightly from $100 \mathrm{~d}$ to about $180 \mathrm{~d}$ of age and increased thereafter. They estimated the heritability of live weight at 150 and $330 \mathrm{~d}$ to be 0.20 and 0.35 , respectively, which is somewhat higher than our estimates but shows the same overall picture of increased heritability with age. The heritability estimates of Bw_330d and Dwg were also similar to estimates by Smith et al. (1989), although they found yearling weight to have a higher heritability than average daily weight gain (0.33 and 0.25, respectively) in Hereford, Angus, and Red Angus. Knights et al. (1984), however, estimated the heritability (SE) of yearling weight in Angus to be 0.49 (0.05). However, large differences in management, production system, and breed make comparison between the latter 2 studies on beef bulls and Norwegian Red difficult. All genetic correlations among the $\mathrm{BW}$ and growth traits were positive (Table 2), although the genetic correlation between Bw_150d and Dwg was not significantly different from zero. The genetic correlation was 0.64 between Bw_150d and Bw_330d, and 0.83 between Bw_330d and Dwg. This is in accordance with Smith et al. (1989), who found a strong genetic correlation between average daily weight gain and yearling weight (0.92). Further, Prribyl et al. (2008) estimated a genetic correlation between BW at $150 \mathrm{~d}$ and $\mathrm{BW}$ at $330 \mathrm{~d}$ of 0.77 .

\section{BW, Growth Traits, and Semen Characteristics}

Both BW traits were favorably genetically correlated with all the semen characteristic traits (Table 3), but with high standard errors for correlations with motility traits and sperm defects. A large amount of data is needed to estimate precise genetic correlations, particularly for traits with low heritability that contain a smaller amount of genetic information. For Bw_150d and $\mathrm{Bw} \_330 \mathrm{~d}$, the highest genetic correlations were with Mot0h (0.76) and Mot48h (0.66), respectively. In contrast to the BW traits, Dwg had negative genetic correlations with Conc $(-0.25)$ and Mot0h $(-0.53)$, although correlations were favorable with the remaining traits. Smith et al. (1989) also found a negative genetic correlation between average daily weight gain and motility $(-0.36)$ for beef bulls and a negative genetic correlation between motility and yearling weight $(-0.36)$. 
Table 2. Estimated variance components and heritability for BW of Norwegian Red bulls when $150 \mathrm{~d}$ (Bw_150d) and $330 \mathrm{~d}$ (Bw_330d) old, and for average daily weight gain (Dwg) from 150 to $330 \mathrm{~d}$, as well as genetic correlations between traits (SE in parentheses)

\begin{tabular}{|c|c|c|c|c|c|}
\hline \multirow[b]{2}{*}{ Trait } & \multicolumn{2}{|c|}{ Variance component ${ }^{1}$} & \multirow[b]{2}{*}{ Heritability } & \multicolumn{2}{|c|}{ Genetic correlation } \\
\hline & $\sigma_{a}^{2}$ & $\sigma_{e}^{2}$ & & Bw_330d & Dwg \\
\hline Bw_150d (kg) & $58.5(17.1)$ & $369.7(16.5)$ & $0.14(0.04)$ & $0.64(0.11)$ & $0.11(0.18)$ \\
\hline Bw_330d (kg) & $183.5(42.3)$ & $526.3(34.6)$ & $0.26(0.06)$ & & $0.83(0.06)$ \\
\hline $\operatorname{Dwg}(g / d)$ & $3,479.2(727.3)$ & $6,812.6(563.0)$ & $0.34(0.06)$ & & \\
\hline
\end{tabular}

${ }^{1}$ Where $\sigma_{a}^{2}$ is the additive genetic variance and $\sigma_{e}^{2}$ is residual variance.

In their study, both birth weight and weaning weight correlated genetically with motility $(0.36$ and 0.13 , respectively). Thus, the genetic correlation between motility and BW was positive for weight measured early in the bull's life and got weaker with increasing age, which corresponds to our results. Knights et al. (1984) also estimated the genetic correlations between BW (weaning and yearling weight) and subjectively scored semen concentration and motility for Angus bulls. In contrast to Smith et al. (1989) and our results, they found the genetic correlation between these semen quality traits and BW traits to increase from weaning until the bulls were 1 yr old (0.30 and 0.75 , respectively). Furthermore, they estimated the genetic correlation between objectively measured concentration $(\mathrm{spz} / \mathrm{mL})$ and yearling weight to be 0.31 , which agrees with our result for the genetic correlation between concentration and Bw_330d of 0.32 . We estimated the genetic correlations between sperm defects and Bw_150d, Bw_330d, and Dwg to be $-0.41,-0.52$, and -0.28 , respectively; hence, higher BW and daily weight gain are associated with fewer sperm defects. This corresponds to Smith et al. (1989), who found positive genetic correlations between percent of normal sperm and weight traits of $0.20,0.26$, and 0.34 for weaning weight, yearling weight, and daily weight gain, respectively. Because of the lack of literature on these genetic correlations in dairy and dual-purpose cattle, we consider these measures in beef to be relevant. It should be noted, however, that all standard errors were high in Smith et al. (1989), and no standard errors were given in Knights et al. (1984).

Our results indicate that the genetic correlations between weight and growth traits and semen characteristics depend on the age of the bulls; that is, they might be different for young and adult bulls. A greater BW at a given age has been associated with lower age of puberty and maturity; in particular, nutrition before 6 mo is known to affect the onset of puberty (Brito et al., 2012). This means that the bulls in our data set with a high BW at $150 \mathrm{~d}$ might be more sexually mature during the andrology test taken at approximately 12 mo age than bulls that started out at a lower weight. Similarly, a bull with a low BW at 150 d can have a high average weight gain but an increased age of pu-

Table 3. Heritability $\left(h^{2}\right)$ of semen characteristics ${ }^{1}$ and genetic correlations between semen characteristics and BW of Norwegian Red bulls when $150 \mathrm{~d}$ (Bw_150d) and $330 \mathrm{~d}$ (Bw_330d) old, and with average daily weight gain (Dwg) in between (SE in parentheses)

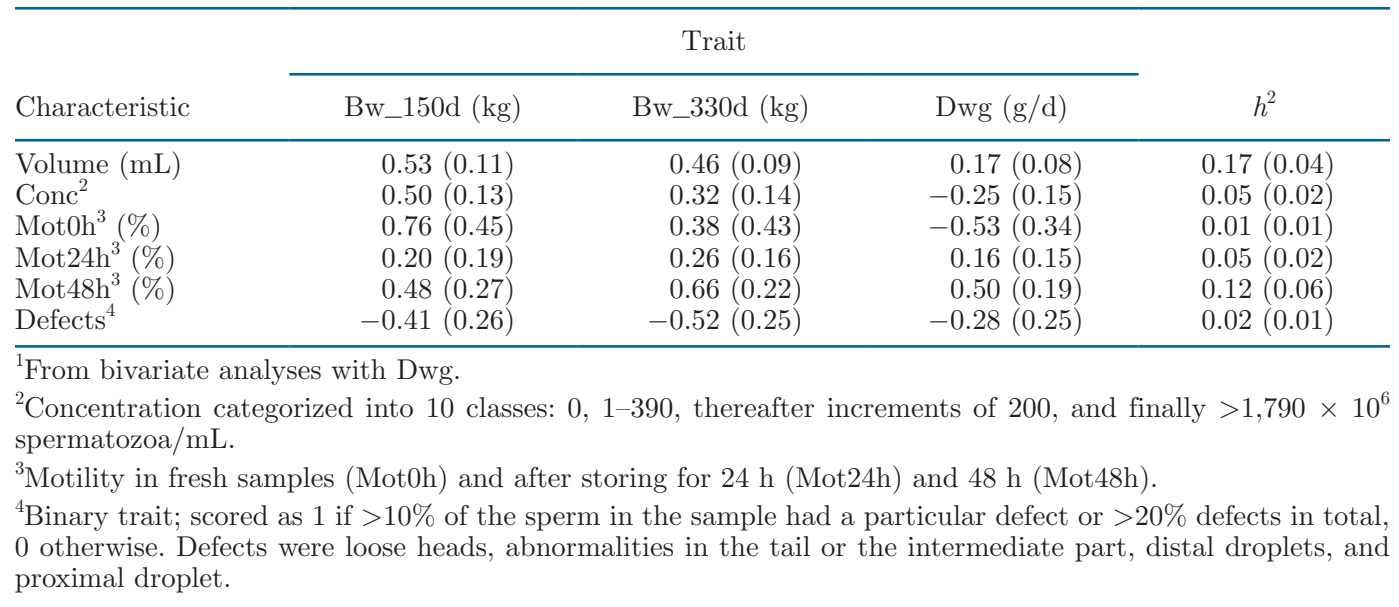


berty. Olsen et al. (2020) suggested that some of the bulls could be sexually immature at the andrology test, which might explain the favorable genetic correlation between the BW traits and all semen characteristic traits, and might explain why the genetic correlations were stronger between Bw_150d and volume, Conc, and Mot0h than between Bw_330d and the aforementioned andrology traits. Furthermore, the negative genetic correlations between Dwg and Conc and Mot0h could reflect the immaturity of bulls at testing for andrology traits.

In addition to our recommendation of making sure bulls are sexually mature and prepared in the same way (Olsen et al., 2020), it would be useful to measure all traits on all semen collections, because Mot24h and especially Mot48h registrations were lacking for many samples in the current data. It is tempting to explain the considerable difference in the genetic correlation for Dwg with Mot0h (-0.53) and Mot48h (0.50), respectively, by different genes affecting the 2 traits. However, another explanation might be differences in the recording of the 2 traits; for example, that only "successful" semen collections, in terms of volume, Conc, or Mot0h, were tested after 24 and $48 \mathrm{~h}$. The strong genetic correlation between Mot0h and Mot24h of 0.96 (Olsen et al., 2020) indicates that the genes affecting the 2 traits are similar and thus points to a difference in recording practice. Consequently, the estimated genetic correlations between Dwg and Mot24h and Mot48h might be closer to expected for properly prepared and sexually mature bulls, but the recording practice might also mean that the phenotypically best bulls receive a measurement of motility after storage. Results from analyses of Mot24h and Mot48h should therefore be interpreted with caution.

\section{Consequences for Selection Response in Semen Characteristics}

Our results suggest that selection on Dwg at the performance test station might have affected sperm Conc and Mot0h negatively, perhaps explaining the slight genetic decline in semen characteristics reported by Olsen et al. (2020). Among volume, Conc and Mot0h, they found the largest relative genetic decline for concentration and the smallest relative genetic change for Mot0h. The attained selection responses are the result of the traits selected for in the performance test, the traits' true genetic parameters, and correlated responses to other traits in the breeding goal. Although selection for Dwg was based on breeding values, selection for semen characteristics has been performed on a phenotypic level by combining the results from the semen collec- tion into an overall score from 0 to 5 , where $\geq 3$ implied that the bull was approved.

\section{CONCLUSIONS}

Our results indicate that the genetic correlations for BW and growth traits with semen characteristics depend on the age of the bulls. Although the majority of genetic correlations were favorable, we found unfavorable genetic correlations between Dwg and Conc and Dwg and Mot0h. Because all genetic correlations among the semen characteristics have been estimated to be favorable, selection for Dwg at the performance test might explain the slight negative genetic trend observed for semen characteristics in young Norwegian Red bulls.

\section{ACKNOWLEDGMENTS}

The authors are grateful to Geno (Hamar, Norway) for access to data, and to the Research Council of Norway (Oslo) for funding (project number: NFR-255097/ E50). The authors have not stated any conflicts of interest.

\section{REFERENCES}

Aass, L. 1996. Variation in carcass and meat quality traits and their relations to growth in dual purpose cattle. Livest. Prod. Sci. 46:112. https://doi.org/10.1016/0301-6226(96)00005-X.

Andersen-Ranberg, I. M., G. Klemetsdal, B. Heringstad, and T. Steine. 2005. Heritabilities, genetic correlations, and genetic change for female fertility and protein yield in Norwegian dairy cattle. J. Dairy Sci. 88:348-355. https://doi.org/10.3168/jds.S0022 -0302(05)72694-1.

Berry, D. P., E. Wall, and J. E. Pryce. 2014. Genetics and genomics of reproductive performance in dairy and beef cattle. Animal 8(S1):105-121. https://doi.org/10.1017/S1751731114000743.

Brito, L. F. C., A. D. Barth, R. E. Wilde, and J. P. Kastelic. 2012. Effect of growth rate from 6 to 16 months of age on sexual development and reproductive function in beef bulls. Theriogenology 77:1398-1405. https://doi.org/10.1016/j.theriogenology.2011.11 .003 .

Carthy, T. R., D. P. Ryan, A. M. Fitzgerald, R. D. Evans, and D. P. Berry. 2015. Genetic parameters of ovarian and uterine reproductive traits in dairy cows. J. Dairy Sci. 98:4095-4106. https://doi .org/10.3168/jds.2014-8924.

Geno. 2018. Total Merit Index. Accessed Dec. 3, 2019. https://www .norwegianred.com/Start/Norwegian-Red/about-norwegian-red/ Norwegian-Red-Total-Merit-Index/.

Knights, S. A., R. L. Baker, D. Gianola, and J. B. Gibb. 1984. Estimates of heritabilities and of genetic and phenotypic correlations among growth and reproductive traits in yearling Angus bulls. J. Anim. Sci. 58:887-893. https://doi.org/10.2527/jas1984.584887x.

Madsen, P., and J. Jensen. 2013. A User's Guide to DMU. A Package for Analysing Multivariate Mixed Models. Version 6, release 5.2. Aarhus University, Aarhus, Denmark.

Olsen, H. B., B. Heringstad, and G. Klemetsdal. 2020. Genetic analysis of semen characteristic traits in young Norwegian Red bulls. J. Dairy Sci. 103:545-555. https://doi.org/10.3168/jds.2019-17291. 
Přibyl, J., H. Krejčová, J. Přibylová, I. Misztal, J. Bohmanová, and M. Stípková. 2008. Trajectory of body weight of performance tested dual-purpose bulls. Czech J. Anim. Sci. 52:315-324. https://doi .org/10.17221/2340-CJAS.

Smith, B. A., J. S. Brinks, and G. V. Richardson. 1989. Estimation of genetic parameters among breeding soundness examination components and growth traits in yearling bulls. J. Anim. Sci. 67:28922896. https://doi.org/10.2527/jas1989.67112892x.

Thundathil, J. C., A. L. Dance, and J. P. Kastelic. 2016. Fertility management of bulls to improve beef cattle productivity. Theriogenology 86:397-405. https://doi.org/10.1016/j.theriogenology.2016.04 .054 .
Tiezzi, F., C. Maltecca, A. Cecchinato, M. Penasa, and G. Bittante. 2012. Genetic parameters for fertility of dairy heifers and cows at different parities and relationships with production traits in first lactation. J. Dairy Sci. 95:7355-7362. https://doi.org/10.3168/jds .2012-5775.

\section{ORCIDS}

H. B. Olsen (®) https://orcid.org/0000-0002-0831-793X

B. Heringstad ๑ https://orcid.org/0000-0001-7388-3893

G. Klemetsdal $\odot$ https://orcid.org/0000-0002-4676-9005 